\title{
Galvanotaxis of Caenorhabditis elegans: current understanding and its application in improving research
}

\author{
Muniesh Muthaiyan Shanmugam* \\ Institute of Molecular and Cellular Biology, National Tsing Hua University, Taiwan (ROC)
}

\begin{abstract}
Electrosensation and movement towards a desired pole in an electric field, electrotaxis or galvanotaxis, is a behavior which is conserved in variety of species from a unicellular organism to multi-cellular organisms. In most cases this behavior is closely related with prey detection, predator detection, host identification by parasite and navigation. Electroreception is widely associated with aquatic organisms, but not restricted to aquatic organisms. Understanding the basic sensory-motor and molecular mechanism behind this behavior in model organisms will provide clues of potential application of this phenomenon. C. elegans has been developed as a simple model organisms to understand basic aspects of biology over several decades. Early analysis of $C$. elegans for electroreception and electrotaxis proved the existence for movement of worms towards the negative electrode in electric field. Following which several research led to improved, but not complete, understanding of the galvanotaxis behavior and mapping of neuronal circuit involved in sensory-motor decision making. Thus, understood behavioral parameters are used in various aspects of worm research such as sorting of worm population for research, analysis of neuro-muscular function of worms in mutants and disease models.
\end{abstract}

\begin{abstract}
Abbreviations
C. elegans - Caenorhabditis elegans; C. briggsae - Caenorhabditis briggsae; EF - electric field; COPAS - Complex object parametric analyzer and sorter; DC - Direct current; AC - Alternating current; 6-OHDA - 6-hydroxy dopamine; MPTP - 1-methyl 4-phenyl 1,2,3,6-tetrahydropyridine.

\section{Introduction}

The behavior of movement of an organism towards a desired direction, when the organism senses an electric field (EF) is known as electrotaxis. This behavior is also called as galvanotaxis. This behavior of electrosensation and subsequent movement towards a direction can be observed in very primitive unicellular organisms, as well as in evolutionary advanced multi-cellular organisms [1-4]. Galvanotaxis behavior is more widely identified in aquatic organisms (e.g. several species of fish), may be evolutionary preserved in aquatic environments as it is an excellent conductor of EF, as well as in few terrestrial organisms (e.g. monotremes, Dictyostelium, cockroaches, bees and nematode worms) [2,5-10]. The predominant use of electrosensation and movement at desired direction is utilized for identification of food such as prey and host, to escape from potential predator and navigation to different locations in an environment.
\end{abstract}

A nematode worm Caenorhabditis elegans (C. elegans) has been developed into an excellent model organism after introduction by Sydney Brenner [11,12]. This model organism is being used to study genetics, development, behavior, neuroscience, physiology, aging, toxicological and host-pathogen interaction. The great advantage of using this worm as model organism is that the worm expresses genes which show $\sim 65 \%$ homology to human genes [13]. The developmental fate of all the cells in C. elegans had been mapped and an adult hermaphrodite possess 302 neurons, making this nematode worm a better model to study genetics and behavior [14]. Further, simple organization of neurons along with available cellular manipulation tools provides a gate-way for understanding some questions related to sensory-motor behavior in this model organism. C. elegans developed simple sensory amphid, phasmid and labial neurons for sensation of chemical, food and temperature, as well as mechanosensory neurons for sensation of touch. Following the identification of environmental cues, the worm has to perform decision making either to move towards the cues or to avoid it [15]. Several events involved in motility of $C$. elegans has been well studied, such as a directional movement of the worm, runs, are interrupted by reorientation events, turn and reversal. If the worm move towards improving desirable sensation then the runs are longer with less turns or reversals, however when moving towards non-improving conditions reorientation events will help to position the worm towards desirable conditions by trial and error method. Further, in certain conditions worms tend to move with prolonged runs without any distractions [16-20]. As a simple and popular model organism $C$. elegans is subject for varieties of behavioral studies and electrotaxis is one among several behaviors exhibited by this nematode model [2].

Understanding any phenomenon in a simple model organism such as C. elegans will greatly help to depict the basic mechanisms behind the observed behavior and to develop numerous research methodologies to improve our understanding, of various aspect of disease and wellbeing, so that the finding can be translated to higher model organisms or for therapeutic uses.

Correspondence to: Muniesh Muthaiyan Shanmugam, Institute of Molecular and Cellular Biology, Department of Life Science, National Tsing Hua University, Taiwan; E-mail: shanmugambms@gmail.com, s100080881@m100.nthu.edu.tw

Key words: Galvanotaxis, electrotaxis, Caenorhabditis elegans, behavior, electrosensation, worms, microfluidics

Received: January 05, 2017; Accepted: January 19, 2017; Published: January 23, 2017 


\section{C. elegans electrotaxis behavior}

The second-half of $20^{\text {th }}$ century introduced galvanotaxis behavior of nematode worms to the scientific community. Bird (1959) showed the attraction of Meloidogyne javanica, a plant parasitic nematode, towards the plant roots is based on the movement of worm along the potential difference caused by the lower redox potential [21]. Following which several articles explained the galvanotaxis phenomenon of nematodes [3,22-24]. C. elegans, despite being used as a good model organism for behavior and development studies, it was only in 1978 galvanotaxis property of C. elegans was studied by Sukul and Croll (1978) by single nematode-tracking techniques. Sukul and Croll (1978) developed different combination of direct current (DC) and potential difference on agar surface with distilled water or $\mathrm{KCl}$ solution and found out that $C$. elegans subjected to either lower or higher voltages and currents moved towards anode and between the voltage of 3 to 4 volts cathodal movement of worms was significant. Their experiments showed that electrotaxis of worm towards electrical poles is a genuine phenomenon which happens as soon as the electricity is turned on and not electrophoresis or kinesis or direct effect of EF on muscle contraction. It can be found that the worms does not reach the electrodes, rather they stopped moving towards the electrode at about $2 \mathrm{~mm}$ before the electrode and engaged in constant reversals. They attributed electrotaxis behavior to the discussion that worms should sense the ionic flow in the electrical fields through their amphids. At lower or high currents, positive charge of metal ions in the electrode or production of oxygen in the anode may attract the worms, respectively. However, cathodal movement may be developed by sensing the steady discharge of potassium and chloride ions [2].

Further, analysis by Klein, Kim and Meyer on the galvanotaxis behavior of worm on agarose gel in $\mathrm{M} 9$ (M9 buffer - $3 \mathrm{~g} \mathrm{KH}_{2} \mathrm{PO}_{4}, 6 \mathrm{~g}$ $\mathrm{Na}_{2} \mathrm{HPO}_{4}, 5 \mathrm{~g} \mathrm{NaCl}, \mathrm{H}_{2} \mathrm{O}$ up to 1 liter, add $1 \mathrm{ml}$ of $1 \mathrm{M} \mathrm{MgSO}_{4}$ after cooldown from sterilization by autoclave [25]) salts showed attraction of worm towards cathode. Here, they showed two different phases of electrotaxis. At relatively lower voltage $(\sim 0.07 \mathrm{~V} / \mathrm{mm})$ the worms showed indiscriminate movement towards cathode, however at higher voltage $(\sim 0.4 \mathrm{~V} / \mathrm{mm})$ the movement towards cathode was more in a directed manner at an angle to the cathode. Occasionally, initial movement of the worm at an angle to cathode is flipped at an angle in the form of letter ' $\mathrm{V}$ ' relative to the starting angle, i.e. the worm moves in a zig-zag pattern toward the cathode. Examination of electrotaxis at different worm stages showed that L4, adult hermaphrodites and males showed the strongest behavioral response, following which L3 and L2 showed weaker and L1 with little or no response to electric field. Among the mutants, they screened to determine the genetic basis of galvanotaxis, some mutants (e.g. lin-32 (u282) and n1754) where found to be electrotaxis defection, (these mutants are also defective of thermal avoidance and chemotaxis). However, $n 1937$ and $n 1938$ mutants which are chemotaxis defective showed wild-type behavior for galvanotaxis. Altogether suggesting a possible involvement of genetic basis as stark relation to electro-sensation behavior ([26], through personal communication).

Gabel et al. (2007) attempted to understand more about the specific genetic and neuronal basis of $C$. elegans galvanotaxis as well as mapped the neuronal circuits involved in this behavior. They also found that the electrotaxis is a robust event, after the worms were subjected to $\mathrm{EF}$ and the worm moves at an angle toward the cathode. However, the angle of approach towards negative electrode increased with the increase in EF strength (Figure 1). Further step-wise rotation of the EF resulted in change in direction of the worm to the new cathode
A

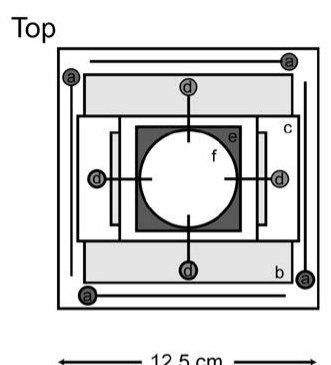

Cross-section

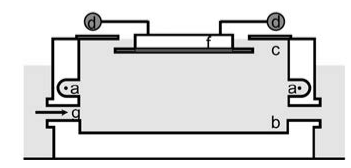

B

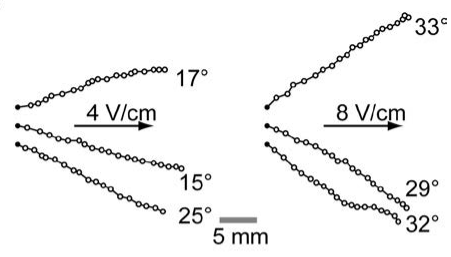

C

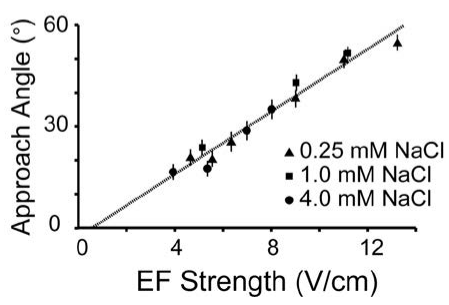

Figure 1. Shows the galvanotaxis setup and the galvanotaxis data from Gabel et al. (2007). A) Shows the galvanotaxis setup from top and cross section with (a \& d) four platinum electrodes, (b) plexiglas chamber with salt solution, (c) a platform to support the agar disc (f) and (e) non-reflective surface beneath the agar disc for dark-field-type illumination by a ring of LEDs (not shown), B) Shows the tracks and the angle of navigation of the wild-type worms at different voltage and C) Shows the relation between the angle of navigation and the EF strength at different salt concentrations. Figure reprinted from Gabel et al. (2007) with permission of Society for Neuroscience [27].

position. Two motility behavior of reorientation maneuvers was observed, first, sudden change in direction of worm without pausing or slowing down, second, backward movement involving reversals following by change in direction of movement. Second behavior was observed when increasing the rotational speed of electric field. Since, the worm is covered by thick cuticle that provides an ionic barrier from the environment and vise-versa, the worm should possess an electrical field sensing mechanism through sensory neurons, which lead to the screening of mutants for defective electro-sensation especially for genes involved in development of sensory organelle, neurotransmission, intracellular transport and calcium sensor. Genes such as che-2, che-13, eat-4,osm-3, osm-5, osm-6, osm-10 and tax-6 are found to be severely defective for galvanotaxis. Further, the neuronal circuit involved in sensing the electrical field and change of direction towards the cathode was mapped by calcium sensing transgenic lines combined with laser ablation of particular neurons inorder to access the contribution of that particular sensory-motor pathway neurons in electro-sensory behavior (Figure 2) [27]. A recent research by Chrisman et al. (2016) explain that the increased trajectory angle of movement towards negative pole at increased field strength is because worms tend to attain a preferred specific EF strength of $\sim 5 \mathrm{~V} / \mathrm{cm}$ when subjected to electric field. Further, they also confirm the important role of AWC neurons' function in galvanotaxis behavior of C. elegans [28].

Analysis of galvanotaxis of C. elegans in a microfluidic device provided further understanding of this behavior, apart from above mentioned electrotactic response by the worm. Microfluidic devices provide better control of the micro-environments using simple devices which regulate physical principles of fluid flow. More information about such devices and use of those devices in C. elegans research can be found elsewhere [29-33]. Different developmental stages of $C$. elegans response to specific range of EF with varied speed of locomotion and sensitivity. The range of effective response for L4 larval stage was found to be 4 to $10 \mathrm{~V} / \mathrm{cm}$ and for that of adult worm was found to be 2 to $4 \mathrm{~V} / \mathrm{cm}$, further, the sensitivity to EF increases with age. Also, above the maximum threshold of EF the worms of particular developmental stage undergo paralysis. Similarly, the speed of motility for adult worm 


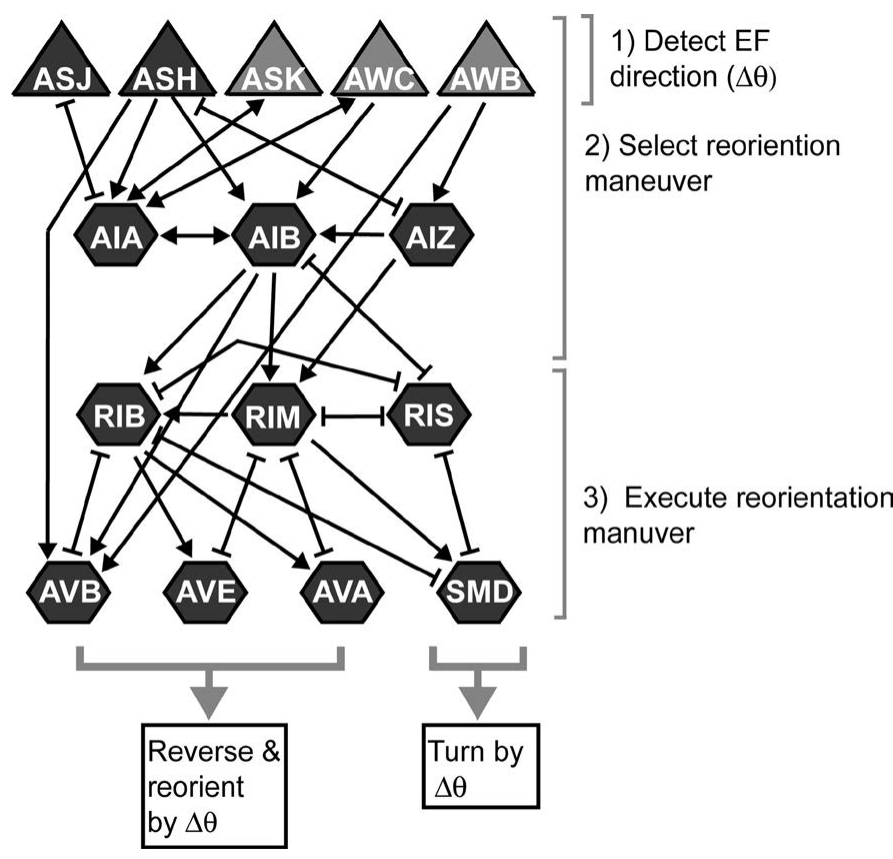

Figure 2. Neural circuit delineated by Gabel et al. (2007) which is involved in sensorymotor galvanotaxis behavior of $C$. elegans. Sensory neurons are showed by triangles, command motor neurons and interneurons are by hexagons, chemical synapses are indicated by arrows and gap junctions by brackets. The primary sensory neurons being ASJ and ASH, whereas RIM and AVA contribute to turns and reversals. 1,2\&3) Shows the activity of neurons in the electrosensory behavior and decision making. Figure reprinted from Gabel et al. (2007) with permission of Society for Neuroscience [27].

is higher when compared to that for L4, whose speed is higher in comparison with L3 larval stage. Worms exhibited normal life cycle, reproductive behavior and locomotion post-exposure to EF and upon post-paralysis events [34]. Rezai et al. (2011) developed a microfluidic device to study the electro-sensation behavior of the worms (C. elegans and $C$. briggsae) (Figure 3) and showed that the worms response not only to constant DC electric field but also to pulse DC electric field [35].

The nematode worm C. elegans is also found to respond to alternating electric current $(\mathrm{AC})$ in a unique pattern, unlike DC electric field, in a microfluidic device. During the positive half of the AC cycle the worms moved towards the cathode, however the direction of the worm was reversed during the negative half of the AC cycle with the frequency of cycle between $\sim 100 \mathrm{mHz}$ to $\sim 1 \mathrm{~Hz}$. The worm either momentarily localized or the speed is greatly reduced during the negative half of the AC cycle. The reversal of the field did not alter the direction of the travel exhibited by the worm. When the worms were subjected to high frequency ranges, between $\sim 1 \mathrm{~Hz}$ to $3 \mathrm{KHz}$, the worms were localized in the microchannel, which may be due to, the time taken by the worm to sense the change in cycle and to initiate the movement is longer when compared to the time taken for change in frequency cycle. Nevertheless, the range of localization differed for worms at different developmental stages. The older animals responded instantaneously by localization in that particular position in the microchannel, whereas L1 and L3 larval staged worms mobilized for a short distance before being localized (for frequency above $1 \mathrm{KHz}$ ) [36].

\section{Application of electrotaxis behavior in worm research}

Electrotaxis behavior of the worm is applied in various aspects of research to understand molecular, cellular and behavioral basics. Following are few examples of application of galvanotaxis in research.

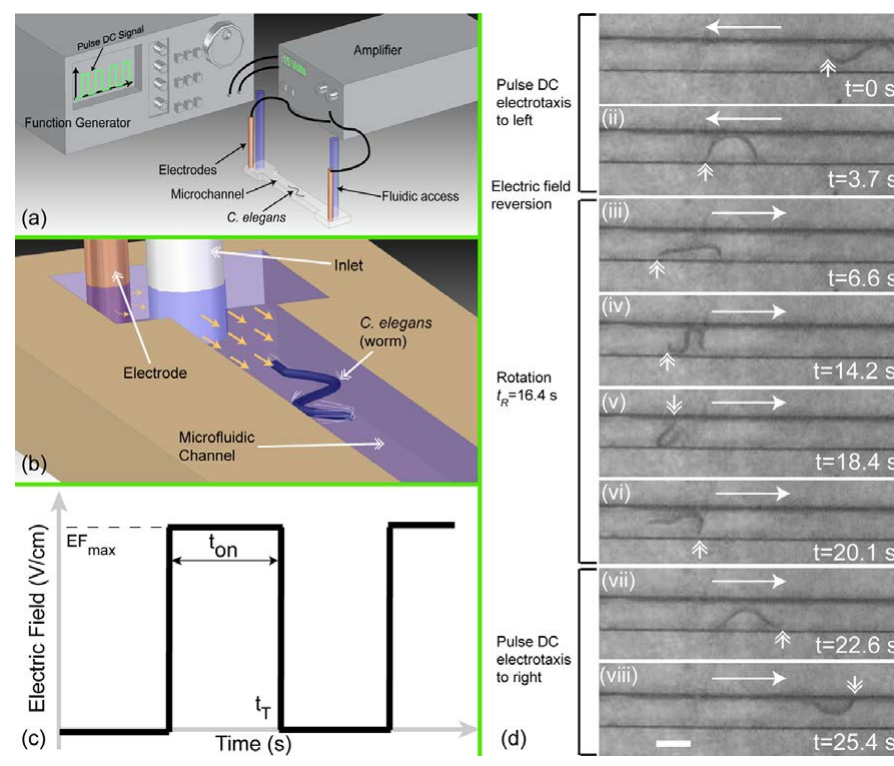

Figure 3. Shows microfluidic chip setup and the worm subject to EF in the device developed by Rezai et al. (2011). a\&b) Shows pictorial representation of the microfluidic device for galvanotaxis studies and the accessory units to regulate the EF, c) Shows the pulse DC EF waveform, d) Shows C. elegans in the microfluidic device which is subjected to electrotaxis and the reversion of the EF led to change of direction of movement. Double headed arrow shows the position of the worm, $\mathrm{EF}_{\max }=3 \mathrm{~V} / \mathrm{cm}$, frequency $=1000 \mathrm{~Hz}$, duty cycle $-30 \%$ and scale bar $=0.3 \mathrm{~mm}$. Figure reprinted from Rezai et al. (2011) with permission of AIP Publishing LLC [35].

C. elegans undergo development through various larval stages to adulthood [37]. Some time, it is necessary to have all the worms of mixed population sorted into a particular developmental stage to address various fundamental questions in research. Despite the availability of various methods to synchronize the worm population by chemical treatment and sorting a worm population from a mixed population by using expensive worm sorter (COPAS from Union Biometrica), use of relatively inexpensive microfluidic devices is a recent developing option. Handful of devices/setup and microfluidic devices had been introduced to scientific community which can sort worms based on electro-sensation behavior of worm into age, size and locomotion defective groups.

Rezai et al. (2012) introduced a microfluidic device that can sort worms using differential electrotactic response of the worm at different age. The microfluidic device created contains an electrical trap with narrow microchannel, which enhances the EF in the narrow channel when compared to the wider channel on either side. This enhanced EF allowed only worms at certain developmental stages to pass through the electrical trap, preventing entry of the worms of other developmental stage, thus sorting the worm of particular population. The working principle is based on the varied electrosensory response of worms to enhanced EF from different developmental stages. Using this device, authors sorted the young adult worms from older worms, young adult worms from larval staged worms and wild-type young adult worms from mutant worms. Although the sorter works at a selectivity of $\sim 90 \%$ for worms of different age and mutants, multi-stage sorting of worms can potentially overcome this issue [38].

Yet another sorting microfluidic device based on combination of electrotactic response and size-dependent motility was introduced by Han et al. (2012). This device has hexagonally designed and arrayed microchannel which allows only a particular size of worm to swim pass the channel and the direction of the swim was regulated with 
electrotaxis phenomenon[39].

A small setup was introduced by Manière et al. (2011) to screen for mutants defective of locomotion and to study the effect of mutation or aging on worm mobility by quantitatively analyzing the worm crawling velocity using galvanotaxis behavior [40].

Salam et al. (2013) used the electrosensory behavior of C. elegans in microfluidic channel to study the behavior of mutants that effect sensory and dopaminergic neurons, thereby proving that electrotaxis assays can be a reliable method to study neuromuscular activity. This study showed that mutant is $35-80 \%$ slower in speed than the wildtype animals. Also, treatment of animals with chemical compounds, such as 6-OHDA, MPTP and rotenone, that are known to induce neurodegeneration and damage to neurons, showed that treated animals reflected similar behavior to mutant animals, whose phenotype can be rescued with pre-exposure of worms with neuroprotective acetaminophen prior to toxic chemical treatment [41].

Chuang et al. (2016) developed a method to analysis the effect of exercise in preventing symptoms of age-related degeneration of neuronal activity in C. elegans using a micro-fluidic platform employing galvanotaxis behavior. By changing the polarity of the EF to which the worm is subjected to, the animal can move constantly to the cathode direction for a set period. This produce a trend-mill effect on the worms, thus employing the worms for exercise [42].

\section{Conclusion}

C. elegans and other nematodes possess ability to sense the EF in a much instantaneous manner [21]. Although, the parasitic nematodes' ability to sense EF can be attributed to identification and interaction with host, it seems irrelevant for C. elegans to display galvanotaxis behavior as soil dueling organism. However, it can be explained that electrotactic response in C. elegans may be evolutionary conserved [27]. In spite of lacking understanding of basic mechanism of electrosensation by sensory neurons in C. elegans, as described above, our current understanding of the behavioral pattern of a simple model organism like C. elegans has resulted in application of this phenomenon to analyze various basic mechanisms of neuromuscular activities and related genes, to analysis the effect of toxic chemical and therapeutic compounds, to understand the benefit of exercise and to sort worm of particular population for research purpose. More understanding in future will open new areas of application, for example: understanding electrotaxis behavior of cultured cells is being translated as a treatment strategy to enhance the wound healing process in humans $[43,44]$.

It can be strongly believed that our understanding of the galvanotaxis, not only in C. elegans but also in other organisms, will increase in times to come. As well as application of galvanotaxis to understand various physiological aspects of living beings will be a new frontier of research and for screening toxic chemical and therapeutic compounds, especially that effect sensory-motor behavior.

\section{References}

1. Adler J, Shi W (1988) Galvanotaxis in bacteria. Cold Spring Harb Symp Quant Biol 53 Pt 1: 23-25. [Crossref]

2. Sukul NC, Croll NA (1978) Influence of potential difference and current on the electrotaxis of Caenorhabditis elegans. Journal of Nematology 10: 314-317. [Crossref]

3. Sukul NC, Das PK, Ghosh SK (1975) Cation-mediated orientation of nematodes under electrical fields. Nematologica 21: 145-150.

4. Fortune ES (2006) The decoding of electrosensory systems. Curr Opin Neurobiol 16: 474-480. [Crossref]
5. Czech-Damal NU, Dehnhardt G, Manger P, Hanke W (2013) Passive electroreception in aquatic mammals. J Comp Physiol A Neuroethol Sens Neural Behav Physiol 199: 555-563. [Crossref]

6. Czech-Damal NU, Liebschner A, Miersch L, Klauer G, Hanke FD, et al. (2012) Electroreception in the Guiana dolphin (Sotalia guianensis). Proc Biol Sci 279: 663668. [Crossref]

7. Pettigrew JD (1999) Electroreception in monotremes. J Exp Biol 202: 1447-1454 [Crossref]

8. Shanley LJ, Walczysko P, Bain M, MacEwan DJ, Zhao M (2006) Influx of extracellular $\mathrm{Ca}^{2+}$ is necessary for electrotaxis in Dictyostelium. J Cell Sci 119: 4741-4748. [Crossref]

9. Newland PL, Hunt E, Sharkh SM, Hama N, Takahata M, et al. (2008) Static electric field detection and behavioural avoidance in cockroaches. J Exp Biol 211: 3682-3690. [Crossref]

10. Clarke D, Whitney H, Sutton G, Robert D (2013) Detection and learning of floral electric fields by bumblebees. Science. 340: 66-69. [Crossref]

11. Brenner S (1973) The genetics of behaviour. British Medical Bulletin 29: 269-271.

12. Riddle DL, Blumenthal T, Meyer BJ, Priess JR (1997) Section I: The biological model. In C. elegans II (second Edn). Cold Spring Harbor Laboratory Press, Cold Spring Harbor, New York.

13. Baumeister R, Ge L (2002) The worm in us - Caenorhabditis elegans as a model of human disease. Trends Biotechnol 20: 147-148. [Crossref]

14. Hobert O (2005) Specification of the nervous system. WormBook, ed. The C. elegans Research Community, WormBook

15. Hart AC (2006) Behavior. WormBook, ed. The C. elegans Research Community, WormBook.

16. Croll NA (1975) Behavioural analysis of nematode movement. Adv Parasitol 13: 71122. [Crossref]

17. Pierce-Shimomura JT, Morse TM, Lockery SR (1999) The fundamental role of pirouettes in Caenorhabditis elegans chemotaxis. J Neurosci 19: 9557-9569. [Crossref]

18. Ryu WS, Samuel AD (2002) Thermotaxis in Caenorhabditis elegans analyzed by measuring responses to defined Thermal stimuli. J Neurosci 22: 5727-5733. [Crossref]

19. Hedgecock EM, Russell RL (1975) Normal and mutant thermotaxis in the nematode Caenorhabditis elegans. Proc Natl Acad Sci U S A 72: 4061-4065. [Crossref]

20. Luo L, Clark DA, Biron D, Mahadevan L, Samuel AD (2006) Sensorimotor control during isothermal tracking in Caenorhabditis elegans. Journal of Experimental Biology 209: 4652-4662. [Crossref]

21. Bird AF (1959) The attractiveness of roots to the plant parasitic nematodes Meloidogyne javanica and M. hapla. Nematologica 4: 322-335.

22. Caveness FE, Panzer JD (1960) Nemic galvanotaxis. Proceedings of the Helminthological Society of Washington 27: 73-74.

23. Croll NA (1970) The behaviour of nematodes: their activity, senses and responses. London: Edward Arnold (Publishers) Ltd pp: ix +117 .

24. Whittaker FH (1969) Galvanotaxis of Pelodera strongyloides (Nematoda: Rhabditidae) Proceedings of the Helminthological Society of Washington 36: 40-42.

25. Stiernagle T (2006) Maintenance of C. elegans. WormBook, ed. The C. elegans Research Community, WormBook.

26. Bob Klein, Stuart Kim, Barbara Meyer, Notes on galvanotaxis. Worm Breeder's Gazette. 11: 64.

27. Gabel CV, Gabel H, Pavlichin D, Kao A, Clark DA, et al. (2007) Neural circuits mediate electrosensory behavior in Caenorhabditis elegans. J Neurosci 27: 7586-7596. [Crossref]

28. Chrisman SD, Waite CB, Scoville AG, Carnell L (2016) C. elegans demonstrates distinct behaviors within a fixed and uniform electric field. PLOS ONE 11: e0151320. [Crossref]

29. Wu H, Odom TW, Chiu DT, Whitesides GM (2003) Fabrication of complex threedimensional microchannel systems in PDMS. J Am Chem Soc 125: 554-559. [Crossref]

30. Zhang M, Wu J, Wang L, Xiao K, Wen W (2010) A simple method for fabricating multilayer PDMS structures for 3D microfluidic chips. Lab Chip 10: 1199-1203. [Crossref]

31. Ren K, Zhou J, Wu H (2013) Materials for microfluidic chip fabrication. Acc Chem Res 46: 2396-2406. [Crossref] 
32. Shanmugam, MM, Santra TS (2016) Microfluidic devices in advances Caenorhabditis elegans research. Molecules 21: 1-16.

33. Shanmugam MM (2016) Improved methods of microinjection in Caenorhabditis elegans: automation and microfluidic systems. JSM Biotechnol Bioeng 3: 1072.

34. Rezai P, Siddiqui A, Selvaganapathy PR, Gupta BP(2010) Electrotaxis of Caenorhabditis elegans in a microfluidic environment. Lab Chip 10: 220-226. [Crossref]

35. Rezai P, Salam S, Selvaganapathy PR, Gupta BP (2011) Effect of pulse direct current signals on electrotactic movement of nematodes Caenorhabditis elegans and Caenorhabditis briggsae. Biomicrofluidics 5: 044116-441169. [Crossref]

36. Rezai P, Siddiqui A, Selvaganapathy PR, Gupta BP (2010) Behavior of Caenorhabditis elegans in alternating electric field and its application to their localization and control. Applied Physics Letters 96: 153702.

37. Altun ZF, Hall DH (2009) Handbook of C. elegans Anatomy. Introduction. In WormAtlas.

38. Rezai P, Salam S, Selvaganapathy PR, Gupta BP (2012) Electrical sorting of Caenorhabditis elegans. Lab Chip 12: 1831-1840. [Crossref]
39. Han B, Kim D, Ko UH, Shin JH (2012) A sorting strategy for C. elegans based on size-dependent motility and electrotaxis in a micro-structured channel. Lab on a Chip 12: 4128-4134. [Crossref]

40. Manière X, Lebois F, Matic I, Ladoux B, Di Meglio JM, et al. (2011) Running worms C. elegans self-sorting by electrotaxis. PLoS One 6: e16637. [Crossref]

41. Salam S, Ansari A, Amon S, Rezai P, Selvaganapathy PR, et al. (2013) A microfluidic phenotype analysis system reveals function of sensory and dopaminergic neuron signaling in C. elegans electrotactic swimming behavior. Worm 2: 24558. [Crossref]

42. Chuang HS, Kuo WJ, Lee CL, Chu IH, Chen CS (2016) Exercise in an electrotactic flow chamber ameliorates age-related degeneration in Caenorhabditis elegans. Sci Rep 6: 28064. [Crossref]

43. Cortese B, Palamà IE, D'Amone S, Gigli G (2014) Influence of electrotaxis on cell behaviour. Integrative Biology 6: 817-830. [Crossref]

44. Kloth LC (2014) Electrical Stimulation Technologies for Wound Healing. Adv Wound Care (New Rochelle) 3: 81-90. [Crossref]

Copyright: $(2017$ Shanmugam MM. This is an open-access article distributed under the terms of the Creative Commons Attribution License, which permits unrestricted use, distribution, and reproduction in any medium, provided the original author and source are credited. 